\title{
On the Effect of Uplink Power Control on Temporal Retransmission Diversity
}

\author{
Rabe Arshad, Laila H. Afify, Hesham ElSawy, Tareq Y. Al-Naffouri, and Mohamed-Slim Alouini
}

\begin{abstract}
Using stochastic geometry, this article studies the retransmission performance in uplink cellular networks with fractional path-loss inversion power control (FPC). We first show that the signal-to-interference-ratio (SIR) is correlated across time, which imposes temporal diversity loss in the retransmission performance. In particular, FPC with lower path-loss compensation factor decreases inter-cell interference but suffers from degraded retransmission diversity. On the other hand, full path-loss inversion achieves almost full temporal diversity (i.e., temporal SIR independence) at the expense of increased inter-cell interference. To this end, the results show that ramping-down the power upon transmission failure improves the overall coverage probability in interference-limited uplink networks.
\end{abstract}

Index Terms-Power Ramping, Retransmission Performance, Stochastic Geometry, Uplink Cellular Networks

\section{INTRODUCTION}

In wireless networks, fading fluctuations and packet transmissions occur at a much shorter time-scale than that of spatial variations in network topology (e.g., due to mobility). Consequently, it is reasonable to consider a spatially-static network topology over sufficiently large number of timeslots [1]-[4]. In such realistic setting, the performance of active links become location dependent due to the spatiotemporal interference correlation. Particularly, the common set of interferers seen by a given receiver leads to temporally correlated aggregate interference [5], which correlates the signalto-interference-ratio (SIR) across time-slots. Furthermore, the common set of interferers seen by proximate receivers leads to spatial interference correlation [5], and hence, spatially correlated SIRs. The concept of the meta distribution has recently evolved to characterize networks where only fading and nodes activity vary over time, which reveals the percentilebased key performance indicators (KPIs) and reflects the location dependent discrepancies among the performance of the coexisting links [1], [2].

While stochastic geometry provides a comprehensive mathematical framework to characterize large-scale networks, the interference spatiotemporal correlations are ignored in the majority of the literature for the simplicity of treatise [6].

Rabe Arshad is with the Electrical and Computer Engineering Department, University of British Columbia, Canada (e-mail: rabearshad@ece.ubc.ca).

Laila Afify is with the School of Communication and Information Technology, Nile University, Cairo, Egypt (e-mail: laila.afify@kaust.edu.sa).

Hesham ElSawy is with the Electrical Engineering Department, King Fahd University of Petroleum and Minerals (KFUPM), Saudi Arabia (e-mail: hesham.elsawy@kfupm.edu.sa).

Tareq Y. Al-Naffouri and Mohamed-Slim Alouini are with King Abdullah University of Science and Technology (KAUST), CEMSE Division, Thuwal, Saudi Arabia (e-mail: \{tareq.alnaffouri, slim.alouini\} @kaust.edu.sa).

A part of this work was done when the first three authors were at KAUST.
However, accounting for the spatiotemporal correlations becomes indispensable when studying spatially and/or temporally proximate events. For instance, the work in [7] shows that the spatial interference correlation between legitimate users and proximate eavesdroppers highly degrades opportunistic secure downlink communications in cellular networks. Drastic diversity loss in MIMO systems are highlighted in [8], [9] due to the inevitable spatial interference correlations across the antenna branches. Retransmission diversity loss is reported in [9]-[11] due to the temporal interference correlations across subsequent time-slots. The negative impacts of spatiotemporal interference correlations are further highlighted in systems that involve simultaneous transmissions to proximate devices and retransmissions over consecutive time-slots such as cooperative relaying networks [12] and cooperative non-orthogonalmultiple-access networks [13]. However, the models in [8][13] focus on either downlink cellular or ad-hoc networks. To the best of the authors' knowledge, the effect of spatiotemporal interference correlation in uplink cellular systems is never addressed in the literature.

This paper characterizes the temporal diversity loss in uplink cellular networks with fractional path-loss inversion power control (FPC). Particularly, we focus on the joint event of successful packet transmission in two consecutive time-slots, where the fractional path-loss compensation factor $\epsilon \in[0,1]$ is varied in the retransmission to improve the overall success probability (i.e., within any of the two time-slots). To this end, we study the effect of FPC on the temporal SIR diversity and assess the impact of varying $\epsilon$ on the overall success probability. The results show that powerdown ramping (i.e., decreasing $\epsilon$ ) strategy is preferable for interference-limited uplink cellular networks, which contrasts the conventionally adopted power-up ramping strategies.

\section{SySTEM MODEL}

We study a single-tier uplink cellular network with singleantenna base stations (BSs) that are modeled via a homogeneous Poisson point process (PPP) $\Psi_{B}$ with intensity $\lambda_{B}$. The users equipment (UEs) point process $\Psi_{u}$ is constructed by uniformly dropping one UE in the Voronoi cell of each BS. ${ }^{1}$ Universal frequency reuse and always active UEs are assumed. We consider the power-law path-loss attenuation model, with exponent $\eta>2$, such that the signal power decays at the rate $d^{-\eta}$ with the distance $d$. A Rayleigh fading environment is considered in which the channel power gains are exponentially distributed with unit mean. Channel gains

\footnotetext{
${ }^{1}$ Due to brevity, we do not include traffic randomness in our analysis. However, it is straight forward to incorporate as shown in [14].
} 
are independent from each other as well as from the spatial locations of the UEs and BSs. The UEs employ FPC with parameter $\epsilon \in[0,1]$ to partially compensate for the path-loss attenuation [15], [16]. The values $\epsilon=0$ and $\epsilon=1$ correspond, respectively, to the constant transmit power and full path-loss inversion. At full path-loss inversion, every UE intends to maintain an average received power of $\rho$ at its serving BS [17]. However, fractional path-loss inversion may be adopted to mitigate prominent interference induced by UEs located faraway from their serving BSs at the expense of maintaining lower average received powers at their serving BSs. Overall, the transmit power of a typical UE to its intended BS at distance $d$ is $P=\rho d^{\eta \epsilon}$. A sufficiently dense network scenario is considered such that every UE can invert the path loss to the serving BS.

\section{Coverage Probability Analysis}

Without loss of generality, we conduct the analysis on a test BS located at an arbitrary origin and denote the location of the test UE as $x_{0}$. For each UE $x_{i} \in\left\{\Psi_{u} \backslash x_{0}\right\}$, let $D_{i}=\min \left\|x_{i}-\Psi_{B}\right\|$ denote the link distance to its serving BS. Due to the correlated sizes of adjacent Voronoi cells, the association link distances $D_{i}$ in adjacent cells are correlated. Further, the set of active interfering UEs in $\Psi_{u}$ is not a PPP. For tractability, the correlations among $D_{i}$ are ignored and the active interfering UEs are approximated via a nonhomogeneous PPP with intensity $\lambda=\lambda_{B}\left(1-e^{-\pi \lambda_{B} r^{2}}\right)$ where $r$ denotes the intended link distance [15].

To study the joint transmission and retransmission performance, we consider a snapshot of two ${ }^{2}$ arbitrary, but consecutive, time-slots over which $\Psi_{B}$ and $\Psi_{u}$ are static and only the channel gains and the transmission/retransmission states of interfering UEs are allowed to change. In compliance with the 3GPP [18], we assume a power ramping scheme such that the UEs change their transmission powers upon transmission failures.

We focus on a two-level power ramping scheme realized by selecting a path-loss compensation factor of $\epsilon_{i}^{(T)}$ for newpacket transmission and $\epsilon_{i}^{(R)}$ for all subsequent retransmissions until transmission success. Power-up and power-down ramping schemes can be realized by choosing $\epsilon_{i}^{(T)}<\epsilon_{i}^{(R)}$ and $\epsilon_{i}^{(T)}>\epsilon_{i}^{(R)}$, respectively. With slight abuse of notation, we use $j \in\{T, R\}$ to denote that a time-slot/user's state in that time-slot is either a new-transmission time-slot/state $(T)$ or a retransmission time-slot/state $(R)$. State transition diagram for transmission/retransmission states is shown in Fig. 1. Let the set $\Psi_{j}=\left\{\left\|x_{i}^{(j)}\right\|, x_{i}^{(j)} \in\left\{\Psi_{u} \backslash x_{0}\right\}\right\}$ contain the distances between the test BS and all interfering UEs operating in the $j^{\text {th }}$ time slot. Then, the SIR at $j^{\text {th }}$ time-slot at the test BS is

$$
\bar{\Upsilon}^{\left(j_{0}\right)}=\frac{P_{0} r_{0}^{-\eta} \tilde{g}_{0}^{\left(j_{0}\right)}}{\sum_{i \in \Psi_{T}} \rho D_{i}^{\eta \epsilon_{i}^{\left(T_{i}\right)}} \tilde{g}_{i}^{\left(T_{i}\right)} r_{i}^{-\eta}+\sum_{i \in \Psi_{R}} \rho D_{i}^{\eta \epsilon_{i}^{\left(R_{i}\right)}} \tilde{g}_{i}^{\left(R_{i}\right)} r_{i}^{-\eta}} \text {, (1) }
$$

where $r_{0}=\left\|x_{0}\right\|$ is the intended link distance, $r_{i}$ represents the distance between the interfering users and the tagged BS, $P_{0}=\rho r_{0}^{\eta \epsilon_{0}^{\left(j_{0}\right)}}$ is the test UE's transmit power, $\epsilon_{0}^{\left(j_{0}\right)}$ is the

\footnotetext{
${ }^{2}$ The extension to multiple time-slots can be done at the expense of more involved expressions.
}

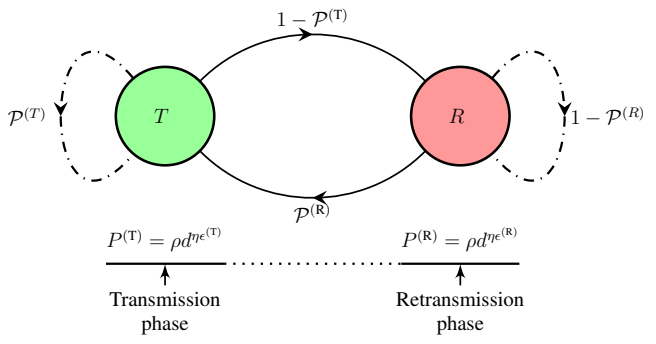

Fig. 1: State transition diagram for the transmission and retransmission states, where $\mathcal{P}^{(j)}$ and $P^{(j)}$ are the transmission success probability and the transmission power at state $j \in\{T, R\}$, respectively.

power control parameter for the test UE at time-slot $j_{0}, \epsilon_{i}^{\left(j_{i}\right)}$ is the power control parameter of the $i^{\text {th }} \mathrm{UE}$ in the $j_{i}^{\text {th }}$ time-slot, and $\tilde{g}_{0}^{\left(j_{0}\right)}$ and $\tilde{g}_{i}^{\left(j_{i}\right)}$ are the intended and interfering channel gains at time-slots $j_{0}$ and $j_{i}$, respectively. For notational convenience, the subscripts for the transmission phases $j$ are dropped hereafter, keeping in mind that the intended link and each of the transmitting links can independently be in either of the $T$ or $R$ phases.

Let $\zeta_{j}$ denote the probability that an interfering UE is employing $\epsilon_{i}^{(j)}$ and $\mathcal{I}^{(j)}$ denote the total aggregate interference when the intended link is at the $j^{\text {th }}$ time-slot. We define the Laplace transforms (LTs) of the marginal and joint probability distribution function (PDF) of the aggregate interference as $\mathcal{L}_{\mathcal{I}}^{(j)}(z) \triangleq \mathbb{E}\left\{\exp \left(-z \mathcal{I}^{(j)}\right)\right\}$ and $\mathcal{L}_{\mathcal{I}}^{(T, R)}\left(z_{T}, z_{R}\right) \triangleq$ $\mathbb{E}\left\{\exp \left(-z_{T} \mathcal{I}^{(T)}-z_{R} \mathcal{I}^{(R)}\right)\right\}$, respectively. Both LTs are characterized via the following lemma.

Lemma 1: The LT of the aggregate interference resulting from the UEs employing FPC is given by

$$
\begin{gathered}
\mathcal{L}_{\mathcal{I}(j)}(z)=\exp \left[-\int_{0}^{\infty} \frac{2 \zeta_{T}\left(\pi \lambda_{B}\right)^{1-\epsilon_{i}^{(T)}}}{\eta \mathrm{w}^{1-\frac{2}{\eta}}\left(1+\frac{\mathrm{w}}{\rho z}\right)} \gamma\left(1+\epsilon_{i}^{(T)}, \pi \lambda_{B} \mathrm{w}^{\left.\frac{2}{\eta\left(1-\epsilon_{i}^{(T)}\right)}\right)}\right) d \mathrm{w}\right. \\
\left.-\int_{0}^{\infty} \frac{2 \zeta_{R}\left(\pi \lambda_{B}\right)^{1-\epsilon_{i}^{(R)}}}{\eta \mathrm{w}^{1-\frac{2}{\eta}}\left(1+\frac{\mathrm{w}}{\rho z}\right)} \gamma\left(1+\epsilon_{i}^{(R)}, \pi \lambda_{B} \mathrm{w}^{\frac{2}{\eta\left(1-\epsilon_{i}^{(R)}\right)}}\right) d \mathrm{w}\right] .
\end{gathered}
$$

Further, the joint LT of interference powers $\mathcal{I}^{(T)}$ and $\mathcal{I}^{(R)}$ is given by (3).

Proof: The proofs of equations (2) and (3) follow from the probability generating functional (PGFL) of PPPs and the moment generating function (MGF) of $\tilde{g}_{i}^{(T)}$ and $\tilde{g}_{i}^{(R)}[10$, Lemma 2] and then averaging over the conditional distance distribution $f_{D_{i} \mid r_{i}}\left(d \mid r_{i}\right)=\frac{2 \pi \lambda_{B} d e^{-\pi \lambda_{B} d^{2}}}{1-e^{-\pi \lambda_{B} r^{2}}}, 0 \leq d \leq r_{i}$. [15].

Assuming fixed transmission rate of $\ln (1+\theta)$ [nats/sec/Hz], the probability of successful packet transmission is defined as $\mathcal{P}^{(j)}=\mathbb{P}\left(\bar{\Upsilon}^{(j)}>\theta\right), j \in\{T, R\}$. Utilizing Lemma 1, we derive the average coverage probability in the next theorem.

Theorem 1: The coverage probability for a single transmission at an arbitrary time instance $j$, for a predefined SIR threshold $\theta$, is given as

$$
\mathcal{P}_{c}^{(j)}(\theta)=\int_{0}^{\infty} 2 \pi \lambda r_{0} e^{-\pi \lambda r_{0}^{2}} \mathcal{L}_{\mathcal{I}}^{(j)}\left(\frac{\theta r_{0}^{\eta\left(1-\epsilon^{(j)}\right)}}{\rho}\right) \mathrm{d} r_{0} .
$$

Further, the average coverage probability within any of the $T$ or $R$ time slots is given by 


$$
\mathcal{L}_{\mathcal{I}}^{(T, R)}\left(z_{T}, z_{R}\right)=\exp \left[-\int_{0}^{\infty}\left[1-\frac{1}{\left(1+\frac{\rho z_{T}}{\mathrm{w}}\right)} \frac{1}{\left(1+\frac{\rho z_{R}}{\mathrm{w}}\right)}\right]\left\{\frac{2 \zeta_{T} \gamma\left(1+\epsilon_{i}^{(T)}, \pi \lambda_{B} \mathrm{w}^{\frac{2}{\eta\left(1-\epsilon_{i}^{(T)}\right)}}\right)}{\eta\left(\pi \lambda_{B}\right)^{\epsilon_{i}^{(T)}}-1 \mathrm{w}^{1-\frac{2}{\eta}}}+\frac{2 \zeta_{R} \gamma\left(1+\epsilon_{i}^{(R)}, \pi \lambda_{B} \mathrm{w}^{\left.\overline{\eta\left(1-\epsilon_{i}^{(R)}\right.}\right)}\right)}{\eta\left(\pi \lambda_{B}\right)^{\epsilon_{i}^{(R)}}-1 \mathrm{w}^{1-\frac{2}{\eta}}}\right\} d \mathrm{w}\right]
$$

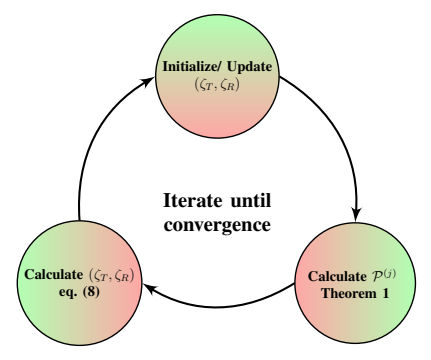

Fig. 2: Solving the system of equations in $\mathcal{P}^{(j)}$ and $\zeta_{j}$

$\mathcal{P}_{c}^{(T, R)}(\theta)=\mathbb{P}\left(\bar{\Upsilon}^{(T)}>\theta\right)+\mathbb{P}\left(\bar{\Upsilon}^{(R)}>\theta\right)-\mathbb{P}\left(\bar{\Upsilon}^{(T)}>\theta, \bar{\Upsilon}^{(R)}>\theta\right)$ where the marginal coverage probabilities are expressed in (4) and the joint coverage probability is given by

$$
\mathbb{P}\left(\bar{\Upsilon}^{(T)}>\theta, \bar{\Upsilon}^{(R)}>\theta\right)=\left.\int_{0}^{\infty} 2 \pi \lambda r_{0} e^{-\pi \lambda r_{0}^{2}} \mathcal{L}_{\mathcal{I}}^{(T, R)}\left(z_{T}, z_{R}\right)\right|_{z_{j}=u_{j}} \mathrm{~d} r_{0}
$$

where $u_{j}=\frac{\theta r_{0}^{\eta\left(1-\epsilon_{o}^{(j)}\right)}}{\rho}$.

Proof: Eq. (4) follows from the complementary cumulative distribution function (CCDF) of the exponential random variable $\tilde{g}_{0}^{(j)}$, and by averaging over the pdf of $r_{0}$ given as $f_{r_{0}}(r)=2 \pi \lambda_{B} r e^{-\pi \lambda_{B} r^{2}}, r_{0}>0$. Eq. (5) follows from the law of total probability. The joint CCDF of $\bar{\Upsilon}^{(T)}$ and $\bar{\Upsilon}^{(R)}$ can be expressed as

$\mathbb{P}\left(\bar{\Upsilon}^{(T)}>\theta, \bar{\Upsilon}^{(R)}>\theta\right)=\mathbb{E}\left[\mathbb{P}\left(\tilde{g}_{0}^{(T)}>\frac{\theta \mathcal{I}^{(T)}}{\rho r_{0}^{\eta\left(\epsilon_{o}^{(T)}-1\right)}}, \tilde{g}_{0}^{(R)}>\frac{\theta \mathcal{I}^{(R)}}{\rho r_{0}^{\eta\left(\epsilon_{o}^{(R)}-1\right)}}\right)\right]$.

Now, incorporating the CCDFs of the i.i.d. $\tilde{g}_{0}^{(T)}$ and $\tilde{g}_{0}^{(R)}$, leads to (6).

The probabilities $\zeta_{j}$ are required to compute the LTs in Lemma 1 and the success probabilities in Theorem 1. According to the two state Markov model in Fig. 1, the steady state probabilities for being in $T$ and $R$ states are given by

$$
\zeta_{T}=\frac{\mathcal{P}_{c}^{(R)}}{1-\mathcal{P}_{c}^{(T)}+\mathcal{P}_{c}^{(R)}} \quad \text { and } \quad \zeta_{R}=\frac{1-\mathcal{P}_{c}^{(T)}}{1-\mathcal{P}_{c}^{(T)}+\mathcal{P}_{c}^{(R)}}
$$

The computation of $\zeta_{j}$ in (7) requires the knowledge of the transmission success probabilities, which introduces an interdependence problem between the success probabilities $\mathcal{P}^{(j)}$ and the steady state probabilities $\zeta_{j}$. Since the system of equations in (4) and (7) cannot be solved in closed form for $\mathcal{P}^{(j)}$ and $\zeta_{j}$, we propose the iterative solution shown in Fig. 2, which converges by virtue of the fixed point theorem. The proof follows [19, Appendix B].

\section{Numerical And Simulations Results}

We first verify the accuracy of the proposed mathematical model via Monte Carlo simulations. Unless otherwise stated, the simulations setup is as follows; the power control parameter $\rho=-50 \mathrm{dBm}$, the path-loss exponent $\eta=4$, and the BSs intensity $\lambda_{B}=4 \mathrm{BSs} / \mathrm{km}^{2}$.

Fig. 3 shows the coverage performance for the single transmission, retransmission with correlated SIRs (i.e., fixed network topology), and retransmission assuming independent SIRs (i.e., independent network topologies) for $\epsilon^{(T)}=\epsilon^{(R)}=\epsilon$. The figure manifests the negative impact of temporal SIR correlation on temporal diversity. The figure also shows the interesting tradeoff, imposed by power control, between the SIR coverage probability and retransmission diversity. Full path-loss inversion makes the SIR coverage probability independent of the UE association distance, which makes the SIRs at the two time slots almost independent (see Fig. 3(a)) but at the expense of increasing the interference level and degrading the coverage probability at high SIR thresholds. Decreasing the path-loss compensation induces more correlations among the SIRs across the two time slots and degrades the temporal diversity, but improves the performance at high SIR threshold due to the decreased interference level in the network as shown in Figs. 3(b) and 3(c).

Fig. 4 shows the effect of power ramping via varying $\epsilon$ upon transmission failure on the correlated success probability $\mathcal{P}^{(T, R)}$. Fig. 4(a) focuses on the power-up ramping scheme with $\epsilon^{(T)}=0.5$ and $\epsilon^{(R)} \geq 0.5$. The figure illustrates that increasing $\epsilon^{(R)}$ deteriorates the coverage performance. Despite that increasing $\epsilon^{(R)}$ improves the temporal diversity, such increase aggravates the aggregate interference induced from UEs in the retransmission phase that deteriorates $\mathcal{P}^{(T, R)}$. Figs. 4(b) and 4(c) provide a closer look into the powerramping performance. Particularly, Fig. 4(b) shows a counterintuitive behavior of improving (degrading) the overall success probability (i.e., $\mathcal{P}^{(T, R)}$ ) when ramping-down (ramping-up) the transmission power in the retransmission phase. This is only true for $\epsilon^{(T)} \geq 0.5$, which is the optimal power control factor in channel inversion based power control [16]. For completeness, we also plot the coverage probability for $\epsilon^{(T)}=0$, which is almost a concave function of $\epsilon^{(R)}$. Note that $\epsilon^{(T)}=0$ does not offer a fair comparison as it does not impose any power control in the uplink.

To further investigate the power ramping behaviour, the explicit success probabilities $\left(\mathcal{P}^{(T)}, \mathcal{P}^{(R)}\right.$, and $\left.\mathcal{P}^{(T, R)}\right)$ are plotted in Fig. 4(c). The figure shows that $\mathcal{P}^{(T)}=\mathcal{P}^{(R)}$ for $\epsilon^{(T)}=\epsilon^{(R)}$ and that $\mathcal{P}^{(T, R)}$ is slightly higher due to the temporal correlation of SIR. Ramping-up the power $\epsilon^{(T)}<\epsilon^{(R)}$ improves the retransmission success at the expense of significant deterioration in $\mathcal{P}^{(T)}$ due to aggravating the aggregate interference. Thus leading to deterioration in the overall success probability as almost $95 \%$ of the UEs go to the retransmission phase. On the other hand, ramping-down the transmission power sacrifices the retransmission success probability but significantly improves the first transmission success probability, which improves the overall success probability. Note that the amount of deterioration in the retransmission performance for the ramping-down scheme is much less than the deterioration of the first transmission performance in the ramping-up scheme. Hence, ramping down strategy is 


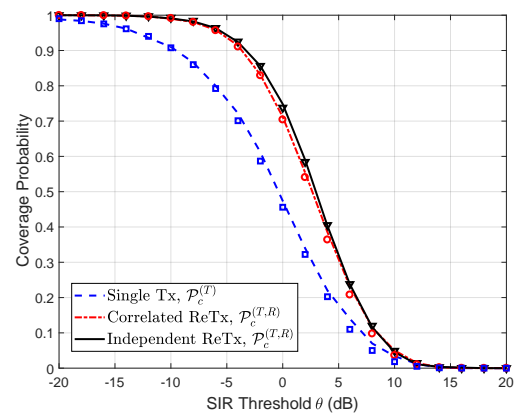

(a) Coverage probability for $\epsilon=1$.

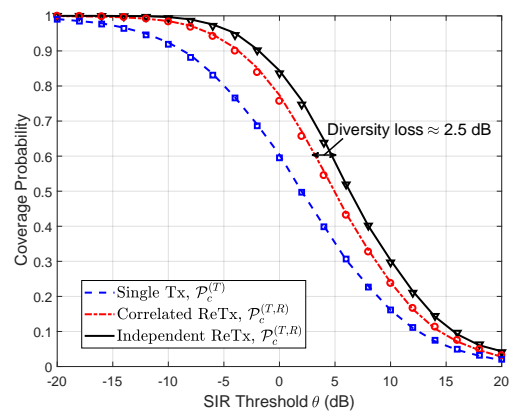

(b) Coverage probability for $\epsilon=0.5$.

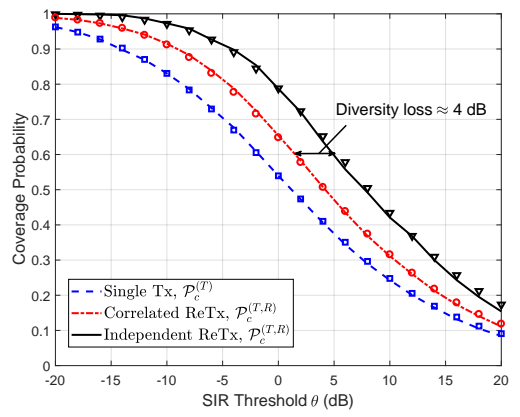

(c) Coverage probability for $\epsilon=0$.

Fig. 3: Theoretical results (lines) and simulations (markers) for the coverage probability at different $\epsilon$ values.

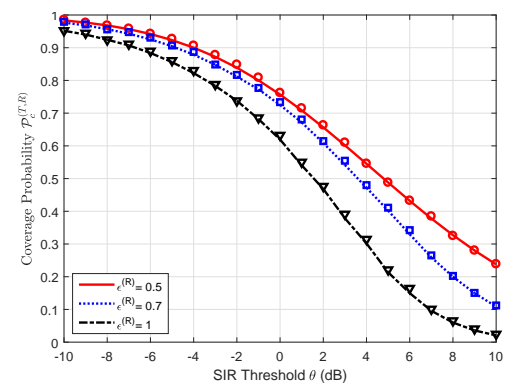

(a) $\epsilon^{(T)}=0.5$ and different values for $\epsilon^{(R)}$.

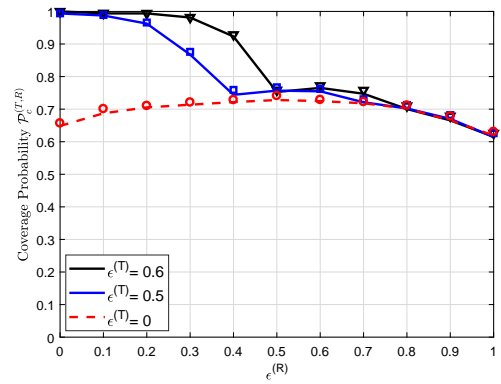

(b) $\theta=0 \mathrm{~dB}$.

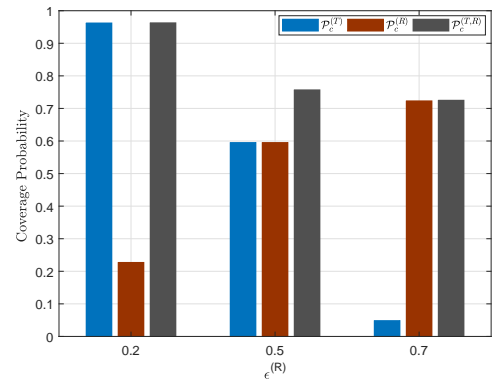

(c) $\epsilon^{(T)}=0.5$ and $\theta=0 \mathrm{~dB}$.

Fig. 4: Coverage probability $\mathcal{P}_{c}^{(T, R)}$ for power ramping schemes with theoretical (lines/bars) and simulations (markers) results.

advocated for interference limited networks to improve the overall success probability.

\section{CONCLUSION}

This paper studies the effect of power control on the temporal correlation of signal-to-interference-ratio (SIR) in uplink cellular networks with fractional path-loss inversion power control. In particular, the paper characterizes the tradeoff imposed by the path-loss compensation factor on the intercell interference and retransmission diversity. The results show that increasing the path-loss compensation factor improves the retransmission diversity at the expense of increasing the intercell interference. Adopting a ramping-up strategy prioritizes retransmissions at the expense of significant deterioration in the first transmission, which diminish the overall success probabilities. To this end, we advocate a power-down ramping strategy that is shown to improve the first transmission as well as the overall success probabilities.

\section{REFERENCES}

[1] M. Haenggi, "The meta distribution of the SIR in Poisson bipolar and cellular networks," IEEE Trans. Wireless Commun., vol. 15, no. 4, pp. 2577-2589, Apr. 2016.

[2] H. ElSawy and M. S. Alouini, "On the meta distribution of coverage probability in uplink cellular networks," IEEE Commun. Lett., vol. 21, no. 7, pp. 1625-1628, July 2017.

[3] M. Gharbieh et al., "Spatiotemporal stochastic modeling of IoT enabled cellular networks: Scalability and stability analysis," IEEE Trans. Commun., vol. 65, no. 99, pp. 3585-3600, Aug 2017.

[4] Y. Zhong et al., "On the stability of static poisson networks under random access," IEEE Transactions on Communications, vol. 64, no. 7, pp. 2985-2998, July 2016.

[5] R. K. Ganti and M. Haenggi, "Spatial and temporal correlation of the interference in ALOHA ad hoc networks," IEEE Commun. Lett., vol. 13, no. 9, pp. 631-633, Sep 2009.
[6] H. ElSawy et al., "Modeling and analysis of cellular networks using stochastic geometry: A tutorial," IEEE Commun. Surveys Tuts., vol. 19, no. 1, pp. 167-203, Firstquarter 2017.

[7] K. S. Ali et al., "The effect of spatial interference correlation and jamming on secrecy in cellular networks," IEEE Wireless Commun. Lett., vol. 6, no. 4, pp. 530-533, Aug 2017.

[8] R. Tanbourgi et al., "Dual-branch MRC receivers under spatial interference correlation and Nakagami fading," IEEE Trans. Commun., vol. 62, no. 6, pp. 1830-1844, June 2014.

[9] M. Haenggi, "Diversity loss due to interference correlation," IEEE Commun. Lett., vol. 16, no. 10, pp. 1600-1603, 2012.

[10] L. Afify et al., "Unified stochastic geometry model for MIMO cellular networks with retransmissions," IEEE Trans. Wireless Commun., vol. 15 , no. 12 , pp. 8595-8609, 2016.

[11] G. Nigam et al., "Spatiotemporal cooperation in heterogeneous cellular networks," IEEE J. Sel. Areas Commun., vol. 33, no. 6, pp. 1253-1265, June 2015.

[12] A. Crismani et al., "Cooperative relaying under spatially and temporally correlated interference," IEEE Trans. Veh. Technol., vol. 64, no. 10, pp. 4655-4669, Oct 2015.

[13] Zheng Shi et al., "Cooperative HARQ assisted NOMA scheme in largescale D2D networks," arXiv preprint arXiv:1707.03945, 2017.

[14] Y. Zhong et al., "Heterogeneous cellular networks with spatio-temporal traffic: Delay analysis and scheduling," IEEE J. Sel. Areas Commun., vol. 35, no. 6, pp. 1373-1386, June 2017.

[15] S. Singh et al., "Joint rate and SINR coverage analysis for decoupled uplink-downlink biased cell associations in hetnets," IEEE Trans. Wireless Commun., vol. 14, no. 10, pp. 5360-5373, Oct 2015.

[16] M. Di Renzo et al., "Stochastic geometry modeling and system-level analysis of uplink heterogeneous cellular networks with multi-antenna base stations," IEEE Trans. Commun., vol. 64, no. 6, pp. 2453-2476, June 2016.

[17] H. ElSawy et al., "On stochastic geometry modeling of cellular uplink transmission with truncated channel inversion power control," IEEE Trans. Wireless Commun., vol. 13, no. 8, pp. 4454-4469, Aug 2014.

[18] 3GPP TS 36.321, "Evolved universal terrestrial radio access (e-utra); medium access control (mac) protocol specification," Release 10, Oct. 2011.

[19] Yong Zhou et al., "Performance analysis of cooperative communication in decentralized wireless networks with unsaturated traffic," IEEE Trans. Wireless Commun., vol. 15, no. 5, pp. 3518-3530, 2016. 